\title{
RESEARCH
}

Open Access

\section{The pathological features and prognoses of intraductal papillary mucinous neoplasm and mucinous cystic neoplasm after surgical resection: a single institution series}

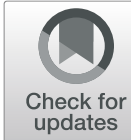

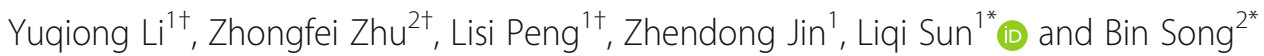

\begin{abstract}
Background: Intraductal papillary mucinous neoplasms (IPMNs) and mucinous cystic neoplasms (MCNs) represent the tumors with malignant transformation potential. The objective of the study was to verify their pathological characteristics, prognoses, and recurrence factors.

Methods: Two hundred eighteen IPMNs and 27 MCNs resected at a single institution were included. The demographic, preoperative, histopathological, and follow-up data of the patients were recorded and analyzed. Overall survival (OS) and disease-free survival (DFS) were defined as the interval from the date of initial surgery to death or the last follow-up (OS) and to diagnosis of recurrence or death at follow-up (DFS).

Results: Of the 218 IPMN and 27 MCN patients, 93 (42.7\%) and 8 (29.6\%) cases were malignant, respectively. IPMNs occurred in older patients compared with MCN patients (median 63 years vs 54 years, $P<0.0001$ ), and MCNs occurred exclusively in females (100\%). Of the overall study cohort, the pathological specimens presented peripheral invasion in 37 (15.1\%) patients and incisal margin invasion was observed in 46 (18.8\%) patients. After a median follow-up of 34 months, 37 (14.9\%) patients relapsed. The 5-year OS and DFS rates of IPMNs were 97.5\% and 80.6\%; and the OS and DFS rates of MCNs were 95.7\% and 87.0\%, respectively. There were four independent risk factors associated with recurrence: pathological diagnoses with malignancy (odds ratio, $O R=3.65)$, presence of oncocytic type for IPMN (OR = 1.69), peripheral invasion $(O R=12.87)$, and incisal margin invasion $(\mathrm{OR}=1.99)$.

Conclusions: IPMNs and MCNs are indolent tumors with favorable prognoses after surgical resection in terms of their relatively high OS and DFS rate. Patients with malignant pathological-related diagnoses should accept strict tumor surveillance in view of their higher risk of recurrence.
\end{abstract}

Keywords: Intraductal papillary mucinous neoplasm (IPMN), Mucinous cystic neoplasm (MCN), Pathological features, Prognosis, Recurrence

\footnotetext{
* Correspondence: zjhzslqsmmu@qq.com; smmusb@126.com

${ }^{\dagger}$ Yuqiong Li, Zhongfei Song, and Lisi Peng are co-first authors of this article.

'Department of Gastroenterology, Changhai Hospital, Navy Medical

University, 168 Changhai Road, Shanghai 200433, China

${ }^{2}$ Department of Hepatobiliary Pancreatic Surgery, Changhai Hospital, Navy

Medical University, 168 Changhai Road, Shanghai 200433, China
}

(c) The Author(s). 2020 Open Access This article is licensed under a Creative Commons Attribution 4.0 International License, which permits use, sharing, adaptation, distribution and reproduction in any medium or format, as long as you give appropriate credit to the original author(s) and the source, provide a link to the Creative Commons licence, and indicate if changes were made. The images or other third party material in this article are included in the article's Creative Commons licence, unless indicated otherwise in a credit line to the material. If material is not included in the article's Creative Commons licence and your intended use is not permitted by statutory regulation or exceeds the permitted use, you will need to obtain permission directly from the copyright holder. To view a copy of this licence, visit http://creativecommons.org/licenses/by/4.0/ The Creative Commons Public Domain Dedication waiver (http://creativecommons.org/publicdomain/zero/1.0/) applies to the data made available in this article, unless otherwise stated in a credit line to the data. 


\section{Introduction}

Cystic neoplasms of the pancreas (PCNs) have a wide clinicopathologic spectrum. Their prevalence ranges from 1.9 to $49.1 \%$ in different races [1-3]. More than half of them are intraductal papillary mucinous neoplasms (IPMNs) [4-6], and 10-45\% are mucinous cystic neoplasms (MCNs) [7, 8]. IPMNs and MCNs represent tumors that have the potential to progress into invasive cancer. They have been classified separately by the World Health Organization since 1996 [9]. However, it was not until 2006 that the diagnostic criteria and terminology of MCN and branch-duct (BD) IPMN were clearly elucidated, and ovarian-type stroma was recognized as a character of MCN [10].

Except for the distinctive ovarian-type stroma MCNs, other types of MCNs generally do not communicate with the pancreatic ductal system and occur almost exclusively in the body and tail regions of the pancreas. MCNs have a tendency to be quite large with a median size of $4-5 \mathrm{~cm}$. MCNs have a strong female preponderance (20:1) and are usually diagnosed in the middle age (fourth and fifth decades) of life [11]. However, IPMNs are generally defined as cystic tumors involving the ductal system. They affect male and female equally between the elder age (sixth and seventh decades) of life and affect the head of the pancreas more often than any other regions of the pancreas [12].

In other words, IPMNs and MCNs are two different pathological types of tumors with different biological behaviors, although they both have a neoplastic mucinproducing epithelial lining. Due to the difficulty of distinguishing IPMN from MCN by preoperative imaging modalities, many guidelines recommend $\mathrm{MCN}$ and IPMN as the same strategies $[10,13]$. The core strategy for the management of MCNs and IPMNs is surgical resection of tumors with imaging high-risk features [14, 15]. However, few literatures focused on the differences in postoperative clinicopathologic features, recurrence rate, survival rate, and recurrence factors between MCNs and IPMNs. As a result, we may be unable to know whether the same postoperative surveillance strategy is appropriate for them.

The goal of the study is to clarify the clinicopathologic features and long-term outcomes following surgical resections of MCNs and IPMNs in a large singleinstitution, pathology confirmed series to date. Their risk factors of postoperative recurrence were also evaluated.

\section{Methods}

\section{Study population}

We conducted a retrospective study at Changhai Hospital affiliated with Navy/second Medical University, one of the largest pancreatic disease research centers in Shanghai, China. The Institutional Review Board of Changhai
Hospital approved the study. Patients who underwent surgical resection from January 2013 to June 2019 for an identified pancreatic cyst and whose final pathological outcomes were identified with MCNs and IPMNs were included in our study. January 2013 was chosen as the start time because the 2012 International Consensus Guidelines influenced our clinical strategy [11]. All the patients underwent contrast-enhanced computed tomography (CT) and/or magnetic resonance imaging (MRI) preoperatively. Endoscopic ultrasound (EUS) was performed in some patients. And at least 12 months of postoperative imaging follow-up was required for the included patients. Exclusion criteria included (1) cases with an undefined grade of dysplasia, (2) specimens obtained from reresections, (3) concomitant other neoplasms on final pathology (e.g., neuroendocrine tumor, cholangiocarcinoma), (4) patients with a postoperative follow-up period $<12$ months, and (5) patients with unavailable imaging or pathological data.

The decision on surgical treatment was made by our multidisciplinary hepato-pancreatobiliary team for the vast majority of patients in accordance with the International Consensus Guideline [10, 11]. The additional surgical indications were high carcinoembryonic antigen (CEA) level and/or positive cytology in cyst fluid obtained by EUS-guided fine needle aspiration (FNA) or endoscopic retrograde pancreatography. The choices of surgical procedures depended on the location, degree, and extent of diseases.

Patient demographics, clinical history, surgical procedure, detailed pathologic characteristics, margin status, peripheral tissue invasion status, progression details, and follow-up details were extracted from the electronic medical records.

\section{Histopathological assessment}

Surgically resected specimens were reviewed under the supervision of at least one senior pathologist specializing in pancreatic pathology.

The surgical procedures consisted of distal pancreatectomy (DP), pancreaticoduodenectomy (PD), total pancreatectomy (TP), central pancreatectomy (CP), and enucleation. The pathologic finding of IPMNs and MCNs were graded as low grade of dysplasia (LGD), intermediate grade of dysplasia (IGD), high grade of dysplasia (HGD), or invasive cancer based on the highest level of dysplasia encountered in the specimens [16]. The patients who had HGD or invasive cancer were considered as malignant, and the patients with other grades of dysplasia were considered as non-invasive. The duct subtypes of IPMNs are main duct (MD) IPMN, BDIPMN, and mixed type (MT) of IPMN. The epithelial subtypes of IPMNs are gastric, intestinal, pancreatobiliary, oncocytic, and mixed type. The margin status was 
defined as negative, non-invasive component (LGD, IGD, PanIN, and invasive cancer $>5 \mathrm{~mm}$ from the incisal margin), or invasive component (invasive cancer $\leq 5$ $\mathrm{mm}$ from the incisal margin). Peripheral tissue invasion status was consisted of perineural invasion, vascular invasion, cancerization of ducts, lymphatic metastasis, common bile duct invasion, peripancreatic fat invasion, and duodenum invasion.

\section{Definition of recurrence}

Patients were examined every 6-12 months after surgery by serum tumor markers and imaging examinations such as CT, MRI, and EUS.

Recurrence was defined as a tumor confirmed by radiology or histology during postoperative follow-up. The sites of recurrence were classified as the residual pancreas (locoregional) and outside the pancreas (extra-pancreatic). The locoregional recurrence was defined as metachronous development of a tumor in the residual pancreas. Extra-pancreatic recurrence was defined as appearance of tumors outside the pancreas, including the peripancreatic areas (local lymph nodes, nerve plexus), lungs, liver, and peritoneal cavity.

\section{Statistical analysis}

The clinical features, histopathological outcomes, and recurrence data were compared between the two groups (IPMNs group and MCNs group). Categorical data were expressed as frequencies (\%) with total observations $(n)$, and the chi-square test or Fisher's exact test was used for comparison between groups. Continuous data were summarized as medians with interquartile ranges (IQR) and were compared between groups using the Student's $t$ or Mann-Whitney $U$ tests.

Overall survival (OS) was defined as the interval from the date of initial surgery to death or the last follow-up. Perioperative deaths were excluded. Disease-free survival (DFS) was defined as the interval from the date of initial surgery to diagnosis of recurrence or death at follow-up. OS and DFS were assessed by the Kaplan-Meier method with log-rank comparisons. A logistic regression model was established to determine the pathological factors associated with recurrence. All statistical analyses were performed with the SPSS software (version 20; IBM Corp, Somers, NY). Statistical significance was set at $P$ values $<0.05$.

\section{Results}

\section{Demographics and clinicopathological features}

From January 2013 to June 2019, 245 patients with pathologically diagnosed IPMNs and MCNs were included in this study. Of the 245 patients, 218 (89\%) were IPMNs and 27 (11\%) were MCNs. Of 218 IPMNs, 125 (57.3\%) were non-invasive, 93 (42.7\%) were malignant, and $148(67.9 \%)$ of them were males. Of 27 MCNs, 19 (70.4\%) were non-invasive, $8(29.6 \%)$ were malignant, and all of them were females. Detailed patient demographics and clinicopathological features are presented in Table 1.

Demographics and clinicopathological feature in IPMNs associated with malignancy included preoperative diabetes mellitus $(P<0.001)$ and resection procedure with PDs $(P=0.04)$. In contrast, resection procedure with DPs was associated with non-invasive IPMNs $(P=$ 0.006). Most of the malignant lesions were pancreaticobiliary and oncocytic epithelial type $(P=0.006)$. More positive margin status (both non-invasive component and invasive component) on pathology were observed in malignant diseases than in non-invasive diseases $(P=$ 0.014 and $<0.0001$ ).

Males were extremely uncommon in our cohort, and all MCN patients included were females. Among them, $10(37 \%)$ were premenopausal and $17(63 \%)$ were peri$(n=4)$ or postmenopausal $(n=13)$. Six $(22.2 \%)$ premenopausal women presented MCNs during or shortly after pregnancy, and $5(29.4 \%)$ peri- or postmenopausal patients presented estrogen-based high-risk tumors. No baseline demographics and pathological findings evaluated in this study were associated with the pathological finding of malignant diseases. However, the cyst size identified by pathological specimen in malignant cases was larger (median $58.5 \mathrm{~mm}$ ) than that in non-invasive cases $(40 \mathrm{~mm})$ in spite of no statistical significance.

The cases of IPMN and MCN were further compared. MCNs were more common in females $(P<0.0001)$, and the patients tended to be younger than IPMNs $(P<$ $0.0001)$. For resection procedures, DPs were more selected in MCN cases $(P<0.0001)$, and PDs were more often selected in IPMN cases $(P<0.0001)$. The peripheral invasion status was comparable in MCN and IPMN cases. However, margin involvement was significantly more in IPMN cases than that in MCN cases, with either non-invasive component $(P=0.018)$ or invasive component $(P=0.003)$.

\section{Recurrence data}

The median follow-up time of the overall cohort was 34 months (interquartile range (IQR), 19-61 months). In total, 37 (15.1\%) patients experienced postoperative disease recurrence, and 7 (2.9\%) patients died (5 cases died of recurrence) during the follow-up period. Among the 37 cases of recurrence, 25 cases developed locoregional recurrence, and 12 cases developed extra-pancreatic recurrence. The median time of recurrence was 8 months (IQR, 5-13.3 months), and the median time of death was 46 months (IQR, 5.5-83 months). Moreover, 36 (97.3\%) of the 37 recurrence cases developed within 2 years. The OS at 1, 3, and 5 years of MCNs and IPMNs combined 


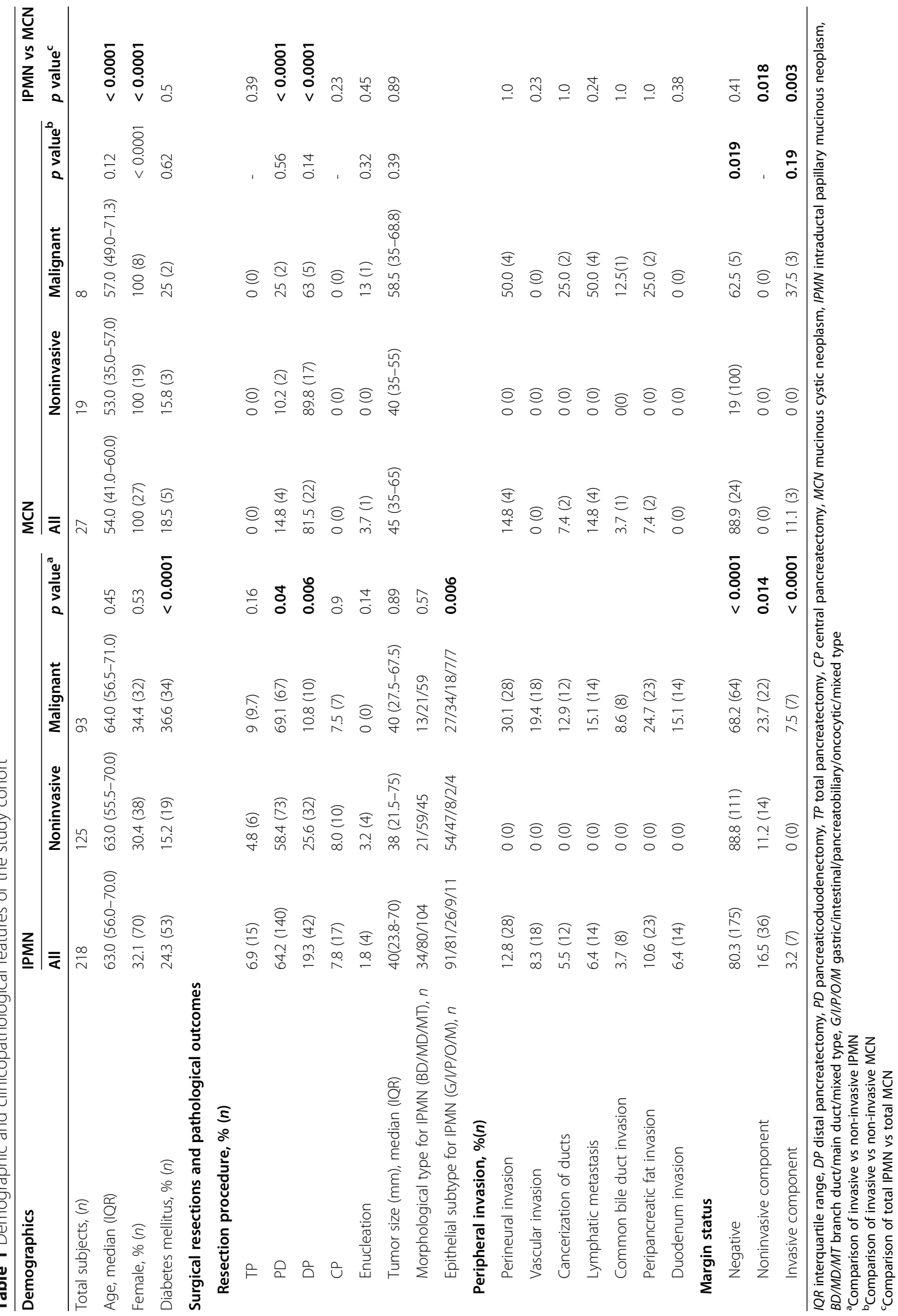


after surgical resection was $98 \%, 95.9 \%$, and $93 \%$, and the DFS was $86.4 \%, 81.8 \%$, and $81.3 \%$, respectively.

For IPMNs, 34 patients $(15.6 \%)$ developed postoperative recurrence, and 6 patients died (4 cases died of recurrence). Of the 93 cases of malignant IPMNs, 22 cases (23.7\%) relapsed and 12 cases $(9.6 \%)$ of 125 non-invasive IPMNs relapsed. The recurrence rate was significantly higher in malignant cases than in non-invasive cases $(P$ $=0.005)$. The OS at 1,3 , and 5 years of IPMNs was $98.75 \%, 98.75 \%$, and $97.5 \%$, and the DFS of IPMNs at 1 , 3 , and 5 years was $85.7 \%, 81.1 \%$, and $80.6 \%$, respectively.

For MCNs, 3 malignant cases of the 27 patients $(11.1 \%)$ developed postoperative recurrence. Only 1 malignant case died after surgical resection due to the extrapancreatic recurrence (omentum majus). No non-invasive cases relapsed or died during the follow-up time. Similarly, the recurrence rate was higher in malignant cases than in non-invasive cases $(P=0.019)$. The OS at 1,3 , and 5 years of MCNs was $95.7 \%, 95.7 \%$, and $95.7 \%$, and the DFS of MCNs at 1, 3, and 5 years was $91.3 \%, 87.0 \%$, and $87.0 \%$, respectively. Detailed recurrence data are presented in Table 2 with corresponding Kaplan-Meier curves in Fig. 1.

According to Kaplan-Meier survival analyses, MCNs and IPMNs had comparable OS and DFS after surgery in overall cohort $(P=0.48$ and 0.67$)$, malignant cases $(P=$ 0.33 and 0.27$)$, and non-invasive cases $(P=1.0$ and 0.2$)$.

\section{Factors associated with recurrence}

Only 3 patients with MCNs relapsed, so to build a logistic regression model for $\mathrm{MCN}$ patients alone was lack of significance. Therefore, we established the logistic regression model of the overall study cohort. In addition, due to the small number of cases in each group, we combined the peripheral invasion status (perineural invasion, vascular invasion, cancerization of ducts, lymphatic metastasis, common bile duct invasion, peripancreatic fat invasion, and duodenum invasion) together, and in total, 37 patients had peripheral invasions. Univariate analysis showed that pathological diagnoses with malignancy $(P<0.0001)$, presence of oncocytic type for IPMN $(P=0.01)$, resection procedure with PD $(P=$ $0.013)$, peripheral invasion $(P<0.0001)$, and incisal margin invasion $(P=0.02)$ were significant pathological risk factors for recurrence. Furthermore, we found four independent risk factors in multivariate analysis: pathological diagnoses with malignancy $(P<0.0001$, odds ratio $(\mathrm{OR})$ $=3.65)$, presence of oncocytic type for IPMN $(P=0.031$, $\mathrm{OR}=1.69)$, peripheral invasion $(P<0.0001, \mathrm{OR}=$ 12.87), and incisal margin invasion $(P=0.039$, OR $=$ 1.99) (Table 3).

\section{Discussion}

This study was conducted in a high-volume pancreatic center with a large cohort. MCNs and IPMNs have the potential of malignant transformation, which is the cause of concern. Surgical resection of them with high-grade dysplasia or invasive cancer has been widely accepted. To determine the optimal high-risk patients for surgical resection, the preoperative imaging risk factor evaluation was recommended by current guidelines [13-15, 17],

Table 2 Summary of results of Kaplan-Meier survival analyses

\begin{tabular}{|c|c|c|c|c|c|c|c|}
\hline & \multirow{2}{*}{$\begin{array}{l}\text { Full cohort } \\
\text { Total }\end{array}$} & \multicolumn{2}{|l|}{ Full cohort } & \multicolumn{2}{|l|}{ Malignant } & \multicolumn{2}{|c|}{ Noninvasive } \\
\hline & & IPMN & MCN & IPMN & MCN & IPMN & MCN \\
\hline \multicolumn{8}{|l|}{ Overall survival } \\
\hline At risk, $(n)$ & 245 & 218 & 27 & 93 & 8 & 125 & 19 \\
\hline Median time, months (IQR) & $34(19-61)$ & $38.5(20-63)$ & $24(16-25)$ & 35 (19-63) & $20(13-23.5)$ & $42(20-64)$ & $20(16-26)$ \\
\hline Deaths, \% (n) & $2.9 \%(7)$ & $2.8 \%(6)$ & $3.7 \%(1)$ & $6.5 \%(6)$ & $12.5 \%(1)$ & 0\% (0) & $0 \%(0)$ \\
\hline 1 -year, \% & $98 \%$ & $98.75 \%$ & $95.7 \%$ & $97.1 \%$ & $87.5 \%$ & $100 \%$ & $100 \%$ \\
\hline 3-year, \% & $95.9 \%$ & $98.75 \%$ & $95.7 \%$ & $94.2 \%$ & $87.5 \%$ & $100 \%$ & $100 \%$ \\
\hline 5-year, \% & $93 \%$ & $97.5 \%$ & $95.7 \%$ & $91.3 \%$ & $87.5 \%$ & $100 \%$ & $100 \%$ \\
\hline Log-rank, $(p)$ & & $p=0.48$ & & $p=0.33$ & & $p=1.0$ & \\
\hline \multicolumn{8}{|l|}{ Disease-free survival } \\
\hline At risk, $(n)$ & 245 & 218 & 27 & 93 & 8 & 125 & 19 \\
\hline Median time, months (IQR) & $24(16-53)$ & $26(17-54)$ & $20(14-25)$ & $23(14-52)$ & $18(8-24)$ & $30(19-58)$ & $20(16-26)$ \\
\hline Recurrence, \% (n) & $15.1 \%(37)$ & $15.6 \%(34)$ & $11.1 \%(3)$ & $23.7 \%(22)$ & $37.5 \%(3)$ & $9.6 \%(12)$ & $0 \%(0)$ \\
\hline 1 -year, \% & $86.4 \%$ & $85.7 \%$ & $91.3 \%$ & $75.3 \%$ & $75.0 \%$ & $94 \%$ & $100 \%$ \\
\hline 3-year, \% & $81.8 \%$ & $81.1 \%$ & $87.0 \%$ & $74.0 \%$ & $62.5 \%$ & $90 \%$ & $100 \%$ \\
\hline 5-year, \% & $81.3 \%$ & $80.6 \%$ & $87.0 \%$ & $71.4 \%$ & $62.5 \%$ & $88 \%$ & $100 \%$ \\
\hline Log-rank, $(p)$ & & $p=0.67$ & & $p=0.27$ & & $p=0.2$ & \\
\hline
\end{tabular}

$I Q R$ interquartile range, $p p$ value, $M C N$ mucinous cystic neoplasm, IPMN intraductal papillary mucinous neoplasm 


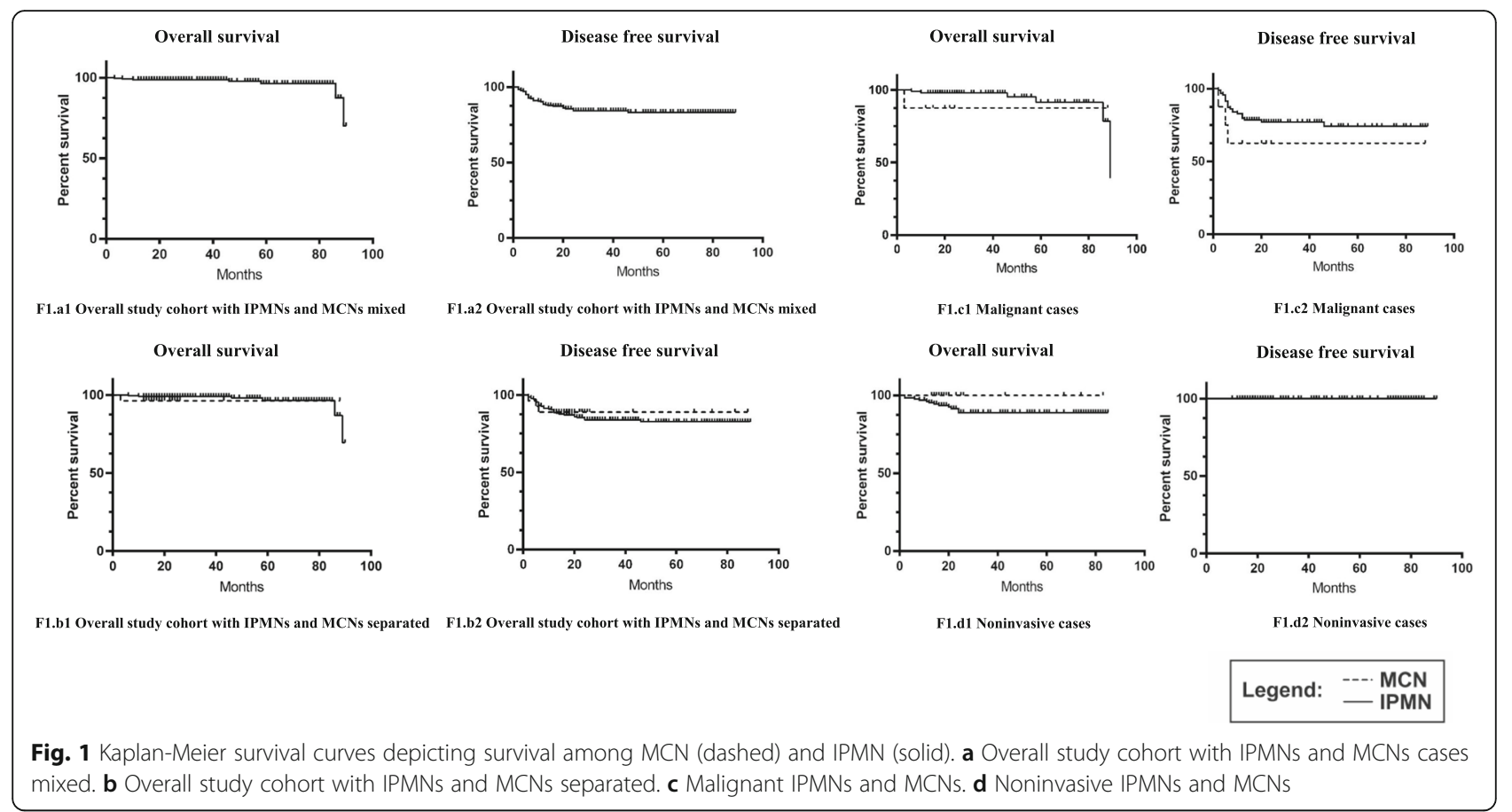

and the evidence level of imaging evaluation was high. Therefore, imaging parameters were not included in our study in order to avoid repetitive work. We focused on the postoperative pathological features, recurrence rate, and recurrence factors of IPMNs and MCNs. Although many studies have discussed the parameters in IPMN [18-21], there are few studies on these issues of MCNs.

The prognosis is favorable for IPMN patients after surgery. A recent study with large cohort conducted by Griffin et al. concluded that IPMN patients after surgery had a 1-year survival rate of $92.7 \%$, and the 5-year survival rate was $72.9 \%$ [22]. Another study by Min et al. concluded that the 5-year disease-specific survival of IPMN was $83.7-100 \%$ and the 5-year recurrence-free survival was 73.1-95\% [23]. A multicenter study of 1074 resected IPMNs in Japan concluded that $14.4 \%$ of patients relapsed [18]. In our study, the 1-year and 5-year OS for IPMN were $98.75 \%$ and $97.5 \%$, and the DFS rate was $85.7 \%$ and $80.6 \%$, respectively. The data in our study were close to the reported data. However, the 5-year OS decreased to $36.0-57.7 \%$ as reported by Griffin et al. [22] for invasive IPMNs, but the data in our study was $91.3 \%$. The bias may be due to the exclusion criteria. In our study, perioperative deaths were excluded. We deemed that these deaths may not reflect the real harm of the diseases. Moreover, the patients in our study were younger (median, 63 years vs 69 years), and the survival curve in our study (Fig. 1) showed an obvious downward trend with the extension of follow-up time. Therefore, the data in our study may not be significantly different from other studies, and prospective studies with the same exclusion criteria are needed to address the problems of real OS and DFS of IPMNs.

MCNs accounted for nearly one fifth of all pancreatic cystic neoplasms. Unlike the more common IPMNs, of which the International Consensus Guidelines in 2006 and 2012 have offered clear criteria for surveillance or resection, surgical resection was recommended for all MCN patients considered candidates for surgery in both guidelines $[10,11]$. Since then, however, many studies have opposed the aggressive recommendation because $\mathrm{MCN}$ is recognized as an indolent tumor. Griffin et al. concluded that MCN had a better prognosis compared to IPMN, and the postoperative 10-year survival rate approached $80 \%$ even in invasive diseases [22]. Postlewait et al. concluded that MCN patients with adenocarcinoma had a 3-year OS and DFS of 59\% and 64\%, respectively [24]. In a multi-institutional, retrospective study conducted by Yamao et al., the 3-, 5-, and 10-year survival rates were $97.6 \%, 96.6 \%$, and $96.6 \%$, respectively [25]. In our study, only 1 in 8 malignant MCN patients died due to the diseases, and the OS and DFS were very similar to the reported data. In summary, $\mathrm{MCN}$ has a favorable prognosis even in malignant cases. Therefore, a more conservative attitude towards surgical resection of MCNs should be adopted.

The characteristic subepithelial tissue of $\mathrm{MCN}$ is ovarian-type stroma. MCN occurs almost exclusively in middle-aged women, and some studies have linked it to pregnancy and hormone-replacement therapy [26-28]. In our study, only 6 patients (22.2\%) developed MCN during or shortly after pregnancy, but it was enough to 


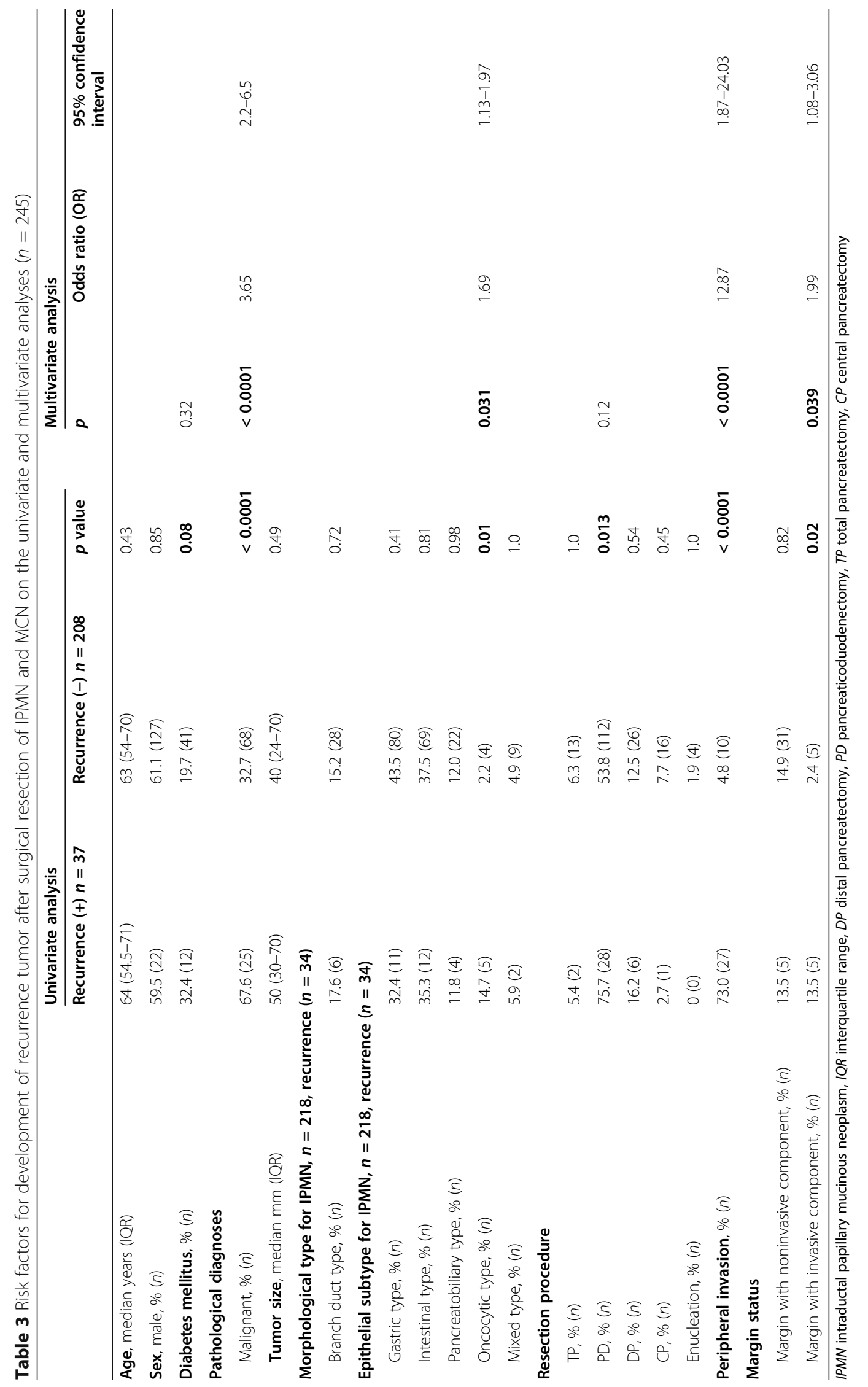


conclude that MCN may be hormonally influenced. The role of hormones in the formation and malignant transformation of MCN needs to be further studied, which may affect the management of $\mathrm{MCN}$ in pregnant and postmenopausal patients.

Many factors have been reported to be associated with tumor recurrence (e.g., symptoms main duct size $\geq 10$ $\mathrm{mm}$, mural nodule size $\geq 5 \mathrm{~mm}$, presence of invasive cancer, lymph node metastasis) $[18,19]$. The recurrence factors identified in our study were pathological diagnoses with malignancy, presence of oncocytic type for IPMN, peripheral invasion, and incisal margin invasion. Although these factors seemed to be diverse, they have one trait in common: they were predictors or characteristic of malignant cases. In other words, malignant cases tended to recur. We should adopt a more active surveillance strategy for those with a malignant pathologicalrelated diagnosis no matter in IPMN or MCN cases. A timely treatment for the recurrent tumor could result in a better prognosis.

However, there was little data to guide the optimal surveillance interval after surgical resection. The latest 2018 American Committee of Gastroenterology guidelines recommended that the surveillance interval should be 6 months for IPMN patients [15]. The surveillance interval of malignant MCNs should follow the guidelines for pancreatic cancer. For benign MCNs, no further surveillance was required [15]. In our study, the median time of recurrence was 8 months (IQR, 5-13.3 months), and recurrence was more common in malignant cases. The data in our study was very similar to the recommendations in the guidelines. Therefore, we believed that it was reasonable to use 6 months as the surveillance interval. In other words, patients with high-risk recurrence factors (diagnosed with malignancy, presence of oncocytic type for IPMN, peripheral invasion, and incisal margin invasion) should undergo regular surveillance every 6 months. The optimal surveillance modalities should be MRI/CT/EUS as we adopted in our study and the guidelines recommended [15].

Our study had several limitations worth discussing. First, it was a retrospective study. The patients were often transferred from other referral institutions. Their initial medical records were not fully presented in our medical system. These patients usually transitioned care closer to the originating hospital following surgery, which also decreased the availability and completeness of long-term and follow-up data collection. The second limitation was the rarity of MCNs, which greatly limited the size of a single institution series. When we designed the study, we tried to build a logistic regression model for MCN to determine which factors were associated with recurrence. However, only $3 \mathrm{MCN}$ patients showed recurrence, which limited the statistical significance of the model.

\section{Conclusions}

This is a large single-institution series of IPMNs and MCNs reported to date with 245 cases confirmed by surgical pathology review. Based on our results, IPMNs and $\mathrm{MCNs}$ had comparable and favorable prognoses after surgical resection. Recurrence occurred in $14.9 \%$ of all the cases. The 5-year OS and DFS for IPMNs approached over $91 \%$ and $71 \%$ even in malignant cases, and the 5-year OS and DFS for malignant MCN cases approached over $87 \%$ and $72 \%$. For those cases without malignancy, OS and DFS approached nearly 100\%. In logistic regression analyses, pathological diagnoses with malignancy, presence of oncocytic type for IPMN, peripheral invasion, and incisal margin invasion were independently associated with tumor recurrence. The results may be beneficial to the identification of IPMN and MCN patients with high risk of postoperative recurrence and further surveillance.

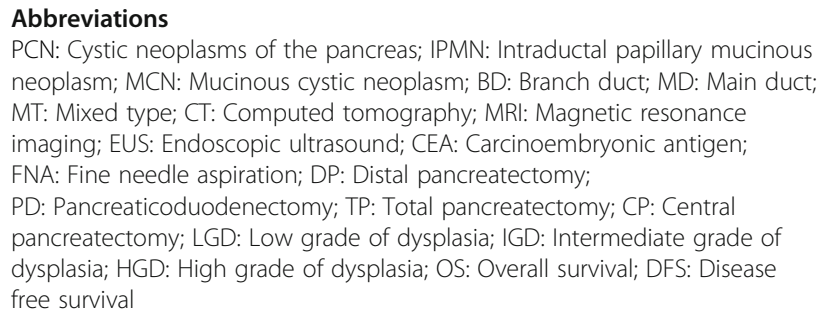

PCN: Cystic neoplasms of the pancreas; IPMN: Intraductal papillary mucinous neoplasm; MCN: Mucinous cystic neoplasm; BD: Branch duct; MD: Main duct; MT: Mixed type; CT: Computed tomography; MRI: Magnetic resonance imaging; EUS: Endoscopic ultrasound; CEA: Carcinoembryonic antigen; FNA: Fine needle aspiration; DP: Distal pancreatectomy; PD: Pancreaticoduodenectomy; TP: Total pancreatectomy; CP: Central pancreatectomy; LGD: Low grade of dysplasia; IGD: Intermediate grade of dysplasia; HGD: High grade of dysplasia; OS: Overall survival; DFS: Disease free survival

\section{Acknowledgements}

We appreciated Haojie Huang and Fang Cui for their kind assistance and advice for this study.

\section{Authors' contributions}

$L Y Q, Z Z F$, and PLS are the main authors of the manuscript and have made substantial contributions to the study design, data acquisition and analysis, and manuscript preparation. JZD has been involved in manuscript editing. SLQ and SB are guarantors of integrity of the entire study and manuscript final version approval. All authors read and approved the final manuscript.

\section{Funding}

This work was supported by the National Natural Science Foundation of China Grant 81502054 (to Bin Song).

\section{Availability of data and materials}

Please contact the corresponding authors for data requests.

\section{Ethics approval and consent to participate}

The study was approved by the Institutional Review Board of Changhai Hospital (Shanghai Changhai Hospital Ethics Committee, reference number: CHEC2019-216).

\section{Consent for publication}

All patients enrolled in the study signed the consent for publication. The written consent is available for review by the Editor-in-Chief of this journal.

Competing interests

The authors declare that they have no competing interests. 
Received: 21 August 2020 Accepted: 21 October 2020

Published online: 04 November 2020

\section{References}

1. Sun L, Wang Y, Jiang F, Qian W, Shao C, Jin Z. Prevalence of pancreatic cystic lesions detected by magnetic resonance imaging in the Chinese population. J Gastroen Hepatol. 2019:34(9):1656-62.

2. Zerboni G, Signoretti M, Crippa S, Falconi M, Arcidiacono PG, Capurso G. Systematic review and meta-analysis: prevalence of incidentally detected pancreatic cystic lesions in asymptomatic individuals. Pancreatology. 2019; 19(1):2-9.

3. Kromrey M, Bülow R, Hübner J, et al. Prospective study on the incidence, prevalence and 5-year pancreatic-related mortality of pancreatic cysts in a population-based study. Gut. 2017;67(1):138-45.

4. Crippa S, Fernandez-Del CC, Salvia R, et al. Mucin-producing neoplasms of the pancreas: an analysis of distinguishing clinical and epidemiologic characteristics. Clin Gastroenterol Hepatol. 2010;8(2):213-9.

5. Salvia R, Malleo G, Marchegiani G, et al. Pancreatic resections for cystic neoplasms: from the surgeon's presumption to the pathologist's reality. Surgery. 2012;152(3 Suppl 1):S135-42.

6. Valsangkar NP, Morales-Oyarvide V, Thayer SP, et al. 851 resected cystic tumors of the pancreas: a 33-year experience at the Massachusetts General Hospital. Surgery. 2012;152(3 Suppl 1):S4-12.

7. Fernandez-del CC, Targarona J, Thayer SP, Rattner DW, Brugge WR, Warshaw AL. Incidental pancreatic cysts: clinicopathologic characteristics and comparison with symptomatic patients. Arch Surg. 2003;138(4):423-7 433-4

8. Brugge WR, Lauwers GY, Sahani D, Fernandez-del CC, Warshaw AL. Cystic neoplasms of the pancreas. N Engl J Med. 2004;351(12):1218-26.

9. Kloppel G, Solicia E, Longnecker D, Capella C, L S. Histologic typing of tumors of the exocrine pancreas: World Health Organization international histologic classification of tumors. 2nd ed. Berlin: Springer-Verlag; 1996.

10. Tanaka M, Chari S, Adsay V, et al. International consensus guidelines for management of intraductal papillary mucinous neoplasms and mucinous cystic neoplasms of the pancreas. Pancreatology. 2006;6(1-2):17-32.

11. Tanaka M, Fernandez-del CC, Adsay V, et al. International Consensus Guidelines 2012 for the management of IPMN and MCN of the pancreas. Pancreatology. 2012;12(3):183-97.

12. Lennon AM, Wolfgang C. Cystic neoplasms of the pancreas. J Gastrointest Surg. 2013;17(4):645-53.

13. Scheiman JM, Hwang JH, Moayyedi P. American gastroenterological association technical review on the diagnosis and management of asymptomatic neoplastic pancreatic cysts. Gastroenterology. 2015;148(4): 824-48

14. European Study Group on Cystic Tumours of the Pancrea. European evidence-based guidelines on pancreatic cystic neoplasms. Gut. 2018;67(5): 789-804.

15. Elta GH, Enestvedt BK, Sauer BG, Lennon AM. ACG clinical guideline: diagnosis and management of pancreatic cysts. Am J Gastroenterol. 2018; 113(4):464-79.

16. Basturk $\mathrm{O}$, Hong SM, Wood LD, et al. A revised classification system and recommendations from the Baltimore Consensus Meeting for neoplastic precursor lesions in the pancreas. Am J Surg Pathol. 2015;39(12):1730-41.

17. Tanaka M, Fernandez-Del CC, Kamisawa T, et al. Revisions of international consensus Fukuoka guidelines for the management of IPMN of the pancreas. Pancreatology. 2017;17(5):738-53.

18. Hirono S, Shimizu Y, Ohtsuka T, et al. Recurrence patterns after surgical resection of intraductal papillary mucinous neoplasm (IPMN) of the pancreas; a multicenter, retrospective study of 1074 IPMN patients by the Japan Pancreas Society. J Gastroenterol. 2020;55(1):86-99.

19. Yogi T, Hijioka S, Imaoka H, et al. Risk factors for postoperative recurrence of intraductal papillary mucinous neoplasms of the pancreas based on a longterm follow-up study: proposals for follow-up strategies. J Hepatobiliary Pancreat Sci. 2015:22(10):757-65.

20. Antonanzas J, Cienfuegos JA, Hurtado-Pardo L, et al. Intraductal papillary mucinous neoplasm (IPMN) of the pancreas: clinicopathological features and long-term outcomes following a pancreatectomy. Rev Esp Enferm Dig. 2018;110(12):768-74.

21. Marchegiani G, Mino-Kenudson M, Sahora $K$, et al. IPMN involving the main pancreatic duct: biology, epidemiology, and long-term outcomes following resection. Ann Surg. 2015;261(5):976-83.
22. Griffin JF, Page AJ, Samaha GJ, et al. Patients with a resected pancreatic mucinous cystic neoplasm have a better prognosis than patients with an intraductal papillary mucinous neoplasm: a large single institution series. Pancreatology. 2017;17(3):490-6.

23. Min JH, Kim YK, Kim H, Cha DL, Ahn S. Prognosis of resected intraductal papillary mucinous neoplasm of the pancreas: using revised 2017 international consensus guidelines. Abdom Radiol (NY). 2020. Epub ahead of print.

24. Postlewait LM, Ethun CG, Mclnnis MR, et al. Association of preoperative risk factors with malignancy in pancreatic mucinous cystic neoplasms: a multicenter study. JAMA Surg. 2017;152(1):19-25.

25. Yamao K, Yanagisawa A, Takahashi K, et al. Clinicopathological features and prognosis of mucinous cystic neoplasm with ovarian-type stroma: a multiinstitutional study of the Japan Pancreas Society. Pancreas. 2011;40(1):67-71.

26. Tanaka S, Kawamura T, Nakamura N, Teramoto K, Arii S. Mucinous cystadenocarcinoma of the pancreas developing during hormone replacement therapy. Dig Dis Sci. 2007;52(5):1326-8.

27. Kosumi K, Takamori H, Hashimoto D, et al. Mucinous cystic neoplasm of the pancreas activated during pregnancy. Surg Case Rep. 2015;1(1):13.

28. Tica AA, Tica OS, Saftoiu A, Camen D, Tica VI. Large pancreatic mucinous cystic neoplasm during pregnancy: what should be done? Gynecol Obstet Invest. 2013;75(2):132-8.

\section{Publisher's Note}

Springer Nature remains neutral with regard to jurisdictional claims in published maps and institutional affiliations.
Ready to submit your research? Choose BMC and benefit from:

- fast, convenient online submission

- thorough peer review by experienced researchers in your field

- rapid publication on acceptance

- support for research data, including large and complex data types

- gold Open Access which fosters wider collaboration and increased citations

- maximum visibility for your research: over $100 \mathrm{M}$ website views per year

At BMC, research is always in progress.

Learn more biomedcentral.com/submissions 